\title{
Observation of Nonlinear Spin-Charge Conversion in the Thin Film of Nominally Centrosymmetric Dirac Semimetal $\mathrm{SrIrO}_{3}$ at Room Temperature
}

\author{
Y. Kozuka $\odot,{ }^{1, *}$ S. Isogami $\odot,{ }^{1}$ K. Masuda $\odot,{ }^{1}$ Y. Miura, ${ }^{1}$ Saikat Das $\odot,{ }^{1}$ J. Fujioka, ${ }^{2}$ T. Ohkubo, ${ }^{1}$ and S. Kasai $\odot^{1,3}$ \\ ${ }^{1}$ Research Center for Magnetic and Spintronic Materials, National Institute for Materials Science (NIMS), \\ 1-2-1 Sengen, Tsukuba 305-0047, Japan \\ ${ }^{2}$ Faculty of Material Science, University of Tsukuba, Tsukuba, Ibaraki 305-8571, Japan \\ ${ }^{3}$ Japan Science and Technology Agency, PRESTO, Kawaguchi, Saitama 332-0012, Japan
}

(Received 17 July 2020; revised 2 December 2020; accepted 7 May 2021; published 9 June 2021)

\begin{abstract}
Spin-charge conversion via spin-orbit interaction is one of the core concepts in the current spintronics research. The efficiency of the interconversion between charge and spin current is estimated based on Berry curvature of Bloch wave function in the linear-response regime. Beyond the linear regime, nonlinear spincharge conversion in the higher-order electric field terms has recently been demonstrated in noncentrosymmetric materials with nontrivial spin texture in the momentum space. Here, we report the observation of the nonlinear charge-spin conversion in a nominally centrosymmetric oxide material $\mathrm{SrIrO}_{3}$ by breaking inversion symmetry at the interface. A large second-order magnetoelectric coefficient is observed at room temperature because of the antisymmetric spin-orbit interaction at the interface of Dirac semimetallic bands, which is subject to the symmetry constraint of the substrates. Our study suggests that nonlinear spin-charge conversion can be induced in many materials with strong spin-orbit interaction at the interface by breaking the local inversion symmetry to give rise to spin splitting in otherwise spin degenerate systems.
\end{abstract}

DOI: 10.1103/PhysRevLett.126.236801

Interconversion of electron spins and charges is one of the central techniques in the current spintronic research. Electrons accelerated by the electric field in a nonmagnetic metal are deflected through spin-orbit interaction, generating spin current transverse to the charge current $[1,2]$. The spin current can be utilized to flip the magnetic moment in the adjacent ferromagnets and applied for electrical magnetization switching with low energy consumption in spintronic devices [3-5]. The efficiency of charge-to-spin conversion has been estimated by calculating spin Hall conductivity based on the Berry phase approach $[1,6]$ or equivalently Kubo formula in the linear-response regime [7]. In this approach, a band degeneracy point acts as a source of Berry curvature, analogous to the magnetic field in the momentum space, and gives a large contribution to spin or anomalous Hall effects $[2,8]$.

Recently, higher-order spin-charge conversion beyond the linear-response theory has been recognized in noncentrosymmetric materials both theoretically and experimentally, where the electric field is coupled with Berry curvature dipole, leading to the observation of second-order nonreciprocal Hall effect [9-15]. Nonlinear spin-charge

Published by the American Physical Society under the terms of the Creative Commons Attribution 4.0 International license. Further distribution of this work must maintain attribution to the author(s) and the published article's title, journal citation, and DOI. conversion is also found to emerge under the in-plane magnetic field for the topological surface states of a topological insulator [16]. This nonlinear planar Hall effect originates from the transverse shift of spin-momentum locked topological surface states under the in-plane magnetic field in the presence of a nontrivial $k$-cubic warping effect. Conversely, it is suggested that the nonlinear planar Hall effect (and nonlinear magnetoresistance as well) can be utilized to probe the nontrivial spin texture in the momentum space in several materials without relying on spin- and angle-resolved photoemission spectroscopy $[16,17]$.

Here, we report a large nonlinear spin-charge conversion detected by a harmonic measurement of the planar Hall effect in nominally centrosymmetric $\mathrm{SrIrO}_{3}$ thin films at room temperature. The orthorhombic phase of $\mathrm{SrIrO}_{3}$ is known to be a Dirac semimetal with comparable spin-orbit interaction and electron correlation $(\sim 0.5 \mathrm{eV})$ with a Dirac nodal ring about $50 \mathrm{meV}$ below the Fermi energy formed from two Dirac bands [18-21]. Owing to the characteristic band structure, this material is known to show a large spin Hall effect and to generate strong spin-orbit torque to the adjacent ferromagnetic layer [22-26]. The crystal structure of $\mathrm{SrIrO}_{3}$ is distorted perovskite $\left(\mathrm{GdFeO}_{3}\right.$-type $)$ in the centrosymmetric $\mathrm{Pbnm}$ space group with lattice constants of $a=5.60 \AA, b=5.58 \AA, c=7.75 \AA$ (corresponding to a pseudocubic lattice constant of $a_{\text {pseudo }}=$ $\sqrt{\left(2 a^{2}+2 b^{2}+c^{2}\right) / 12}=3.93 \AA$ [Fig. 1(a)] [27]. In the 
presence of inversion symmetry, the band structure of $\mathrm{SrIrO}_{3}$ maintains spin degeneracy, and nonlinear spincharge conversion is not expected. However, the spatial inversion symmetry of thin films is inherently broken at the surface and the interface, which causes spin textures in the momentum space due to antisymmetric spin-orbit interaction. Comparing the nonlinear planar Hall effect of $\mathrm{SrIrO}_{3}$ thin films grown on different substrates, we conclude that the observations are interface- or surface-driven phenomena irrespective of the degree of the strain with minor modification of spin texture by the symmetry constraint of the substrate.

The experimental details are explained in the Supplemental Material [28]. Briefly, the $\mathrm{SrIrO}_{3}$ thin films are grown at $650^{\circ} \mathrm{C}$ under 100 mTorr oxygen partial pressure by pulsed laser deposition. We have employed $\quad\left(\mathrm{LaAlO}_{3}\right)_{0.3}\left(\mathrm{Sr}_{2} \mathrm{AlTaO}_{6}\right)_{0.7} \quad$ (LSAT) (001) (cubic, $a=3.87 \AA$ ), $\quad \mathrm{GdScO}_{3} \quad(110)$ (orthorhombic, $a_{\text {pseudo }}=3.96 \AA$ ), and $\mathrm{NdGaO}_{3}$ (110) (orthorhombic, $a_{\text {pseudo }}=3.86 \AA$ ) substrates to compare effects of epitaxial

(a)

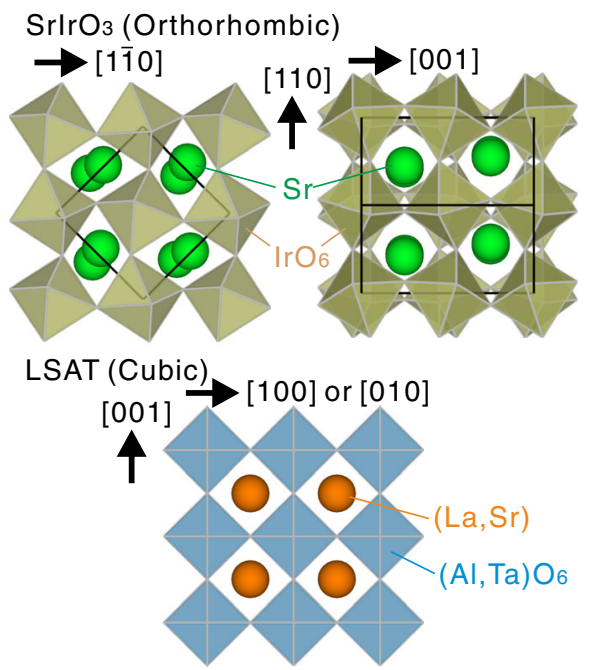

(b)

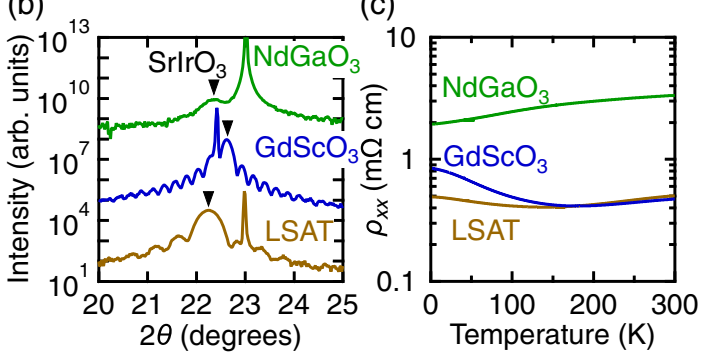

FIG. 1. (a) Schematic diagram of crystal structures for (top) distorted orthorhombic perovskite $\left(\mathrm{SrIrO}_{3}\right)$ and (bottom) cubic perovskite (LSAT) materials. $\mathrm{GdScO}_{3}$ and $\mathrm{NdGaO}_{3}$ have the orthorhombic structure but with different lattice parameters. Black lines in the orthorhombic structure indicate a unit cell. (b) $\theta-2 \theta$ scan of x-ray diffraction and (c) temperature dependence of resistivity for $\mathrm{SrIrO}_{3}$ thin films grown on LSAT (001), $\mathrm{GdScO}_{3}$ (110), and $\mathrm{NdGaO}_{3}$ (110). The triangles in (b) show peaks of $\mathrm{SrIrO}_{3}$ thin films. strain originating from lattice mismatch and crystal symmetry. The thicknesses are 25,27 , and $37 \mathrm{~nm}$ for thin films grown on LSAT (001), $\mathrm{GdScO}_{3}$ (110), and $\mathrm{NdGaO}_{3}$ (110), respectively. The crystal structures of $\mathrm{SrIrO}_{3}$ and LSAT are depicted in Fig. 1(a) [29]. The second-order term of the planar Hall effect is characterized by an out-of-phase second-harmonic component using lock-in amplifiers with an alternating current frequency of $33 \mathrm{~Hz}$ [17]. The firstprinciples density-functional calculations including the spin-orbit interaction are carried out with the aid of the Vienna $a b$ initio simulation program (VASP) [30-32].

Figure 1(b) shows a $\theta-2 \theta$ scan of x-ray diffraction for $\mathrm{SrIrO}_{3}$ thin films grown on LSAT (001), $\mathrm{GdScO}_{3}$ (110), and $\mathrm{NdGaO}_{3}$ (110) substrates, indicating epitaxial growth with out-of-plane lattice constants of $3.99 \AA$ for LSAT, $3.89 \AA$ for $\mathrm{GdScO}_{3}$, and $3.97 \AA$ for $\mathrm{NdGaO}_{3}$, which deviate from the bulk value to compensate in-plane compressive (for LSAT and $\mathrm{NdGaO}_{3}$ ) and tensile (for $\mathrm{GdScO}_{3}$ ) strains (reciprocal space mapping shown in Fig. S1). The crystal structure is additionally characterized by high-angle annular dark-field transmission electron microscope images as shown in Fig. S2. While all films show clear epitaxial growth on the substrates, dislocations are partly observed for LSAT and $\mathrm{NdGaO}_{3}$ substrates probably due to large lattice mismatch and presence of twins. The resistivity shown in Fig. 1(c) is only weakly temperature dependent as typical semimetallic behavior of $\mathrm{SrIrO}_{3}$, consistent with other PLD-grown films in previous reports [33-35].

To characterize the nonlinear planar Hall effect, we measure angular dependence $(\varphi)$ of second-harmonic Hall voltage on in-plane magnetic field direction with respect to the current direction using a Hall bar structure as
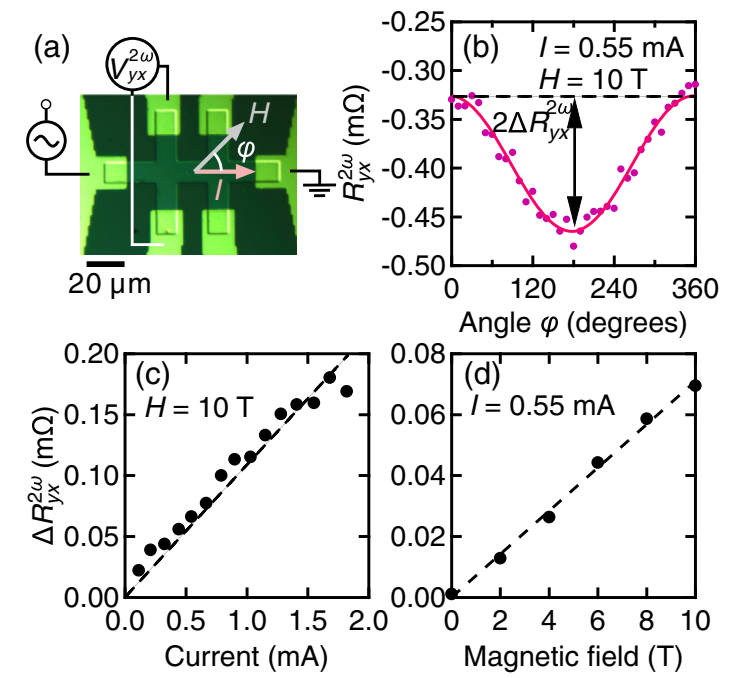

FIG. 2. (a) Microscope image of a Hall bar with $\mathrm{Ti} / \mathrm{Au}$ electrodes together with the measurement geometry. The angle between the current $(I)$ direction and in-plane magnetic field $(H)$ is defined as $\varphi$. (b) Second-harmonic planar Hall resistance $R_{y x}^{2 \omega}=V_{y x}^{2 \omega} / I$ as a function of $\varphi$ and fitting with $\Delta R_{y x}^{2 \omega} \cos \varphi$. (c) Current and (d) magnetic field dependences of $\Delta R_{y x}^{2 \omega}$. 
shown in Fig. 2(a). Figure 2(b) shows a typical secondharmonic planar Hall measurement as a function of $\varphi$ for a film grown on an LSAT substrate at $300 \mathrm{~K}$ under a constant magnetic field $(H)$ of $10 \mathrm{~T}$. The second-harmonic resistance is defined as $R_{y x}^{2 \omega}=V_{y x}^{2 \omega} / I$, where $V_{x y}^{2 \omega}$ is the out-of-phase second-harmonic voltage and $I$ is the externally applied alternating current. The data in Fig. 2(b) clearly show $\cos \varphi$ dependence. So far, a similar cosine dependence of the second-harmonic planar Hall effect has been ascribed to nontrivially spin-momentum locked band structures in noncentrosymmetric materials [17] although, at first sight, this mechanism is not compatible with centrosymmetric $\mathrm{SrIrO}_{3}$.

In order to obtain an insight into the origin, the magnitude of the second-harmonic planar Hall signal $\left(\Delta R_{y x}^{2 \omega}\right)$ is extracted by fitting with $R_{y x}^{2 \omega}=\Delta R_{y x}^{2 \omega} \cos \varphi$ (after subtracting a constant background) [17]. First, as shown in Fig. S3(a) (Supplemental Material [28]), we confirm that $\Delta R_{y x}^{2 \omega}$ is proportional to alternating current [Fig. 2(c)], which indicates the second-harmonic voltage $V_{y x}^{2 \omega}$ certainly captures a second-order signal proportional to $I^{2}$. Subsequently, we investigate magnetic field dependence [Fig. S3(b) of the Supplemental Material [28] ] and find that $\Delta R_{y x}^{2 \omega}$ is proportional to $H$ [Fig. 2(d)]. These results are consistent with the nonlinear planar Hall effect, which is proportional to both the electric field $\left(E_{x}\right)$ and magnetic field $(H)$ as previously observed in $\mathrm{Bi}_{2} \mathrm{Se}_{3}$ [16,17]. We have also confirmed the second-harmonic component of anisotropic magnetoresistance $\left(R_{x x}^{2 \omega}\right)$ as shown in Fig. S4. The ratio between transverse and longitudinal non linear magnetoelectric effect $\rho_{y x}^{2 \omega} / \rho_{x x}^{2 \omega}=\left(R_{y x}^{2 \omega} / R_{x x}^{2 \omega}\right)(L / W)$ ( $L$, channel length $=25 \mu \mathrm{m} ; W$, channel width $=10 \mu \mathrm{m})$ is estimated as 0.50 , and the contribution from the Nernst effect, proportional to $\boldsymbol{H} \times \nabla T$, can be ignored.

The bilinear magnetoelectric effect is known to capture complex spin-momentum locked band structures including warping effect or electron-hole asymmetry. The band structure of bulk $\mathrm{SrIrO}_{3}$ is composed of two Dirac bands around the $U$ point $(\pi, 0, \pi)$ and massive holelike bands around $(0,0,0)$ and $(\pi, \pi, \pi)$ in the Brillouin zone (in the representation of the orthorhombic phase), both of which are spin degenerate due to the centrosymmetric crystal structure $[19,20]$. In the case of thin film, however, antisymmetric spin-orbit interaction induces momentum-dependent spin splitting at the interface, leading to complex spin texture in the two Dirac bands. Indeed, the nonlinear planar Hall effect was also observed at the $\mathrm{LaAlO}_{3} / \mathrm{SrTiO}_{3}$ interface [17] which is known to possess complex spin texture originating from the anticrossing of three $t_{2 g}$ bands $[36,37]$.

To test this consideration, we compare the secondharmonic planar Hall signal for $\mathrm{SrIrO}_{3}$ thin films grown on $\mathrm{NdGaO}_{3}$ (110) and $\mathrm{GdScO}_{3}(110)$ substrates as well as a film grown on LSAT (001) substrate [the raw data of the nonlinear planar Hall effect for $\mathrm{GdScO}_{3}$ with $I \|[001]$ are shown in Figs. S3(c)-S3(f)]. Figure 3 shows the

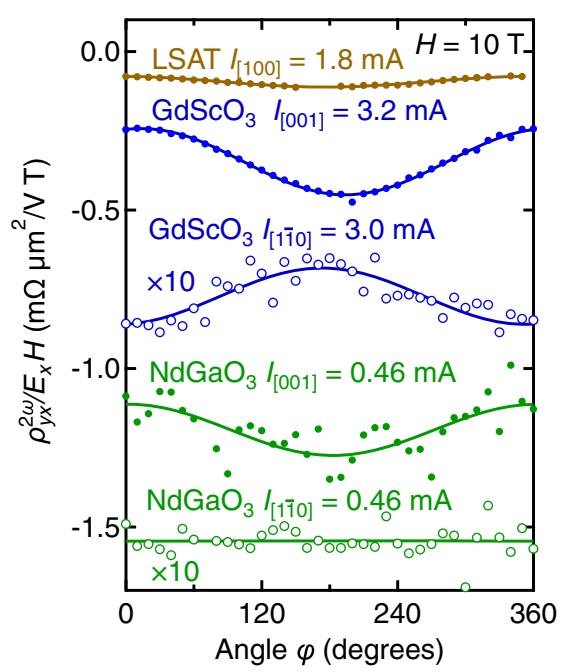

FIG. 3. Second-harmonic planar Hall resistivity $\rho_{y x}^{2 \omega}(=$ $R_{y x}^{2 \omega} d, d$ : thickness) normalized by electric field along the current direction $\left(E_{x}\right)$ and in-plane magnetic field $(H)$ as a function of $\varphi$ for $\mathrm{SrIrO}_{3}$ films grown on three kinds of substrates. For orthorhombic substrates $\left(\mathrm{GdScO}_{3}\right.$ and $\left.\mathrm{NdScO}_{3}\right)$, measurements with two current directions are shown. The data for $\mathrm{GdScO}_{3}$ and $\mathrm{NdGaO}_{3}$ with the current along the [1 10$]$ directions are magnified by a factor of ten because of the small signal.

$\varphi$ dependence of $\rho_{y x}^{2 \omega}$ normalized by $E_{x}$ and $H$, where $\rho_{y x}^{2 \omega}=R_{y x}^{2 \omega} d$ is the second-harmonic resistivity in the threedimensional unit ( $d$, thickness of the film). The magnitudes of the second-harmonic signal remarkably vary with different substrates. Furthermore, in the case of orthorhombic $\mathrm{NdGaO}_{3}$ and $\mathrm{GdScO}_{3}$ substrates, we find a significantly large anisotropy between two current directions along [001] and $[1 \overline{1} 0]$. For fair comparison for films with different resistivity, we use a coefficient of the bilinear magnetoelectric effect $\chi_{y x x}=\Delta \rho_{y x}^{2 \omega} / E_{x} H$, where $\Delta \rho_{y x}^{2 \omega}=\Delta R_{y x}^{2 \omega} d$, which is shown in Table I [17]. We find that the room temperature $\chi_{y x x}$ value for the sample grown on LSAT is almost comparable to the previously reported value for a $\mathrm{Bi}_{2} \mathrm{Se}_{3}$ thin film at $5 \mathrm{~K}\left(\chi_{y x x} \approx 0.02 \mathrm{~m} \Omega \mu \mathrm{m}^{2} / \mathrm{VT}\right)$, which rapidly decreases with increasing temperature toward room temperature. Remarkably, $\chi_{y x x}$ is significantly large for orthorhombic $\mathrm{GdScO}_{3}$ and $\mathrm{NdGaO}_{3}$ with the current along [001] but negligibly small along [1 $1 \overline{1} 0]$. Furthermore, the sign of $\chi_{y x x}$ differs between two current directions in the case of the $\mathrm{GdScO}_{3}$ (110) substrate. These results indicate that the nonlinear planar Hall signal is robust and immune to strain and degree of disorder. The magnitude is sensitive to symmetry constraints from the substrates with minor modulation by the degree of strain, suggestive of an intrinsic effect reflecting the symmetry of the spin texture on the Fermi surfaces. For comparison, we also study a $\mathrm{SrRuO}_{3}$ film grown on LSAT (001) substrate, which does not exhibit nonlinear planar Hall effect at room temperature as shown in Fig. S6. This signals the significance of the strong spin-orbit interaction and the characteristic band structure of $\mathrm{SrIrO}_{3}$. 
TABLE I. Comparison of resistivity and a coefficient of the nonlinear planar Hall effect $\chi_{y x x}=\Delta \rho_{y x}^{2 \omega} / E_{x} H$ for $\mathrm{SrIrO}_{3}$ films grown on various substrates. The data for two distinct current directions are shown for orthorhombic $\mathrm{GdScO}_{3}$ and $\mathrm{NdGaO}_{3}$ substrates.

\begin{tabular}{lccc}
\hline \hline Substrate & $\begin{array}{c}\text { Current } \\
\text { direction }\end{array}$ & $\rho_{x x}(\mathrm{~m} \Omega \mathrm{cm})$ & $\chi_{y x x}\left(\mathrm{~m} \Omega \mu \mathrm{m}^{2} / \mathrm{VT}\right)$ \\
\hline LSAT (001) & {$[100]$} & 0.50 & $0.017 \pm 0.005$ \\
$\mathrm{GdScO}_{3}(110)$ & {$[001]$} & 0.47 & $0.10 \pm 0.004$ \\
& {$[1 \overline{1} 0]$} & 0.52 & $-0.009 \pm 0.001$ \\
$\mathrm{NdGaO}_{3}(110)$ & {$[001]$} & 3.3 & $0.093 \pm 0.01$ \\
& {$[1 \overline{1} 0]$} & 3.3 & $0.0 \pm 0.0001$ \\
\hline \hline
\end{tabular}

In the previous studies, band structure modification in $\mathrm{SrIrO}_{3}$ thin films has been discussed in terms of epitaxial strain and geometric confinement, which causes an enhancement of spin Hall effect in the linear-response regime owing to preferable redistribution of Berry curvature [22,25]. In the present study, the large anisotropy of the nonlinear planar Hall effect reflects the spin-momentum locked Fermi surface composed of two correlated Dirac bands. Since the Fermi surface is deformed to the direction transverse to the external current in the planar Hall geometry with $I \| H$ [17], the large $\chi_{y x x}$ with $I \|[001]$ indicates that spin-momentum locking is stronger along [1피 than along [001]. Since [1 10$]$ is not equivalent to [110] in the orthorhombic structure, this anisotropic spinmomentum locking might be because of the lower in-plane symmetry along [1 10$]$ than along [001], which is crystallographically equivalent to [001] [Fig. 1(a)]. On the contrary, such anisotropy is not present in the case of the cubic LSAT substrate but an orthorhombic twin structure is known to appear based on the detailed structural analysis of $\mathrm{SrIrO}_{3}$ thin films reported in [38]. This twin structure may average out the anisotropic nonlinear planar Hall signal in the case of the LSAT substrate.

To confirm this hypothesis, we have performed firstprinciples calculations of electronic band structure using an unstrained $\mathrm{SrIrO}_{3}$ slab model [Fig. 4(a)] (bulk and strained case are shown in the Supplemental Material [28] for comparison). The band structure is shown in Figs. 4(b) and 4(c). As shown in Fig. 4(c) (magnification around $\bar{U}$ ), inner Ir layers contribute to bands somewhat above the Fermi level, which resembles bulk Dirac bands, whereas the surface top and bottom Ir layers forming Fermi surfaces are expected to be subject to symmetry breaking. To obtain an insight into the spin-resolved band structure around the $\bar{U}$ point [Fig. S7(d)], Fermi surfaces are drawn together with local spins at each $k$ point for the unstrained slab model, projected to Ir atoms in the top layer as shown in Fig. 4(d). The spin texture is found as the surface states originating from the top Ir layer of the slab but summing the spins over the Fermi surfaces exactly compensates the spins, reflecting nonmagnetic nature. Moreover, the
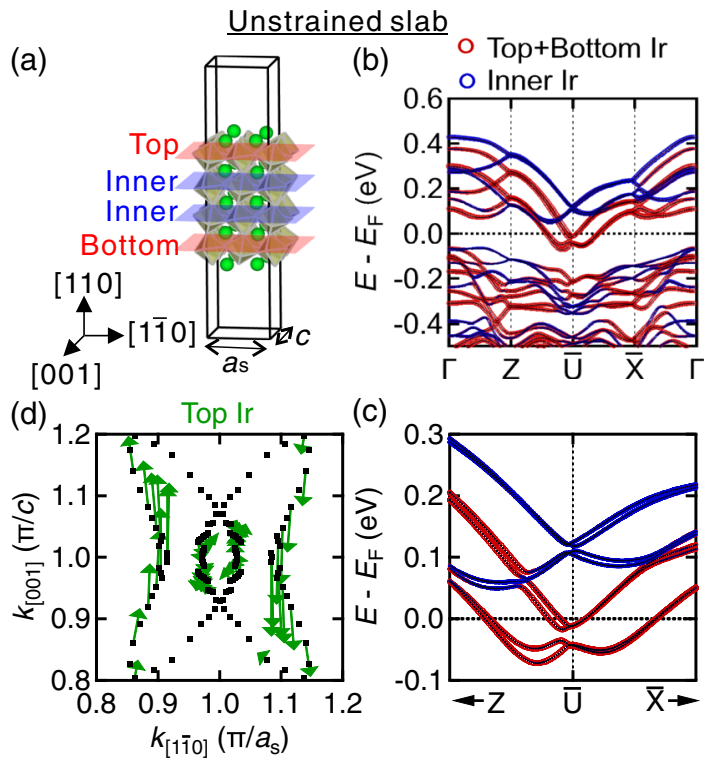

(c)

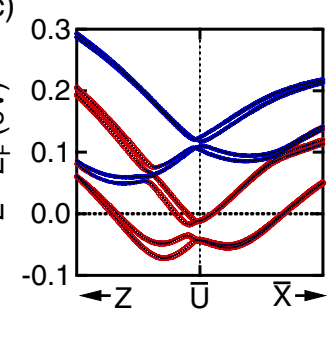

FIG. 4. (a) Crystal structure and (b) band structure of the unstrained slab model. (c) The band structure is magnified around $\bar{U}$. In the panes (b) and (c), contributions from the surface (top and bottom) Ir layers (red) and from inner Ir layers (blue) are separately drawn by color. (d) Fermi surfaces and spin textures around the $\bar{U}$ point, where the contribution from top Ir layers is projected. Comparisons with bulk and strained slab are shown in the Supplemental Material in detail [28].

direction of the spins is inverted between the orbitals from the top and bottom Ir layers [Fig. S8(d)] at each $k$ point. These features are reminiscent of spin structures in the presence of antisymmetric spin-orbit interaction. Compared with conventional semiconductors, spin texture in $\mathrm{SrIrO}_{3}$ is much more complex because spin-orbit interaction plays an essential role in the ground state of the electronic states as represented by the existence of Dirac points.

The obtained spin texture can be compared with the nonlinear planar Hall effect in Fig. 3. In the case of the unstrained slab model, the larger spins are found along [110], while spins are nearly canceled along [001] direction as shown in Fig. 4(d). Since the nonlinear planar Hall effect detects the spin texture orthogonal to the current direction, the larger signal with $I \|[001]$ is consistent with the calculated spin texture. When the $\mathrm{SrIrO}_{3}$ thin film is strained to $\mathrm{GdScO}_{3}$ (110) substrate [39], the Fermi surfaces are enlarged because of the stretched $\mathrm{Ir}-\mathrm{O}-\mathrm{Ir}$ bonds as shown in Figs. S8(e)-S8(h), but the qualitative features are similar to the unstrained case although the spin texture becomes more complex than the unstrained case. This may be consistent with the quantitatively similar magnitude and symmetry of $\chi_{y x x}$ between films grown on $\mathrm{GdScO}_{3}$ and $\mathrm{NdGaO}_{3}$ despite the large difference in strain.

Finally, we remark on the contribution from disorder. In Fig. S9, we show the temperature dependence of the magnitude of the nonlinear planar Hall effect for the $\mathrm{SrIrO}_{3}$ film grown on LSAT (001) substrate. The nonlinear planar 
Hall signal increases with decreasing temperature, exhibiting nearly the same trend as mobility shown in Fig. S9(c), which would indicate disorder origin. However, recent theoretical studies showed that, unlike the spin Hall and anomalous Hall effects in the linear-response regime, the nonlinear Hall effect follows the dependence linear in scattering time $\tau$ both in the intrinsic and the extrinsic cases, which makes it difficult to differentiate these contributions [40,41]. As the nonlinear planar Hall effect [17] has to do with the nonlinear Hall effect observed at zero magnetic field $[14,15]$ in terms of Berry phase dipole in the intrinsic case, we expect that both phenomena may also be similarly influenced by the disorder. As suggested in Ref. [41], precise disorder tuning would be necessary to separate these contributions.

In conclusion, we have observed the nonlinear planar Hall effect in nominally centrosymmetric $\mathrm{SrIrO}_{3}$ thin films at room temperature, which is expected only in noncentrosymmetric systems with nontrivial spin textures in the momentum space. Comparing the nonlinear planar Hall signal between $\mathrm{SrIrO}_{3}$ films grown on different substrates, we conclude that the observation originates from complex the spin texture in the two Dirac bands triggered by the antisymmetric spin-orbit interaction. The strength, sign, and anisotropy of the nonlinear planar Hall signal are modulated by the in-plane symmetry of the substrates, which may sensitively reflect anisotropy of the spin texture of the Dirac bands. Our study suggests that the nonlinear planar Hall effect can be observed in more diverse materials with strong spin-orbit interaction by engineering spin texture in the momentum space with utilizing epitaxial strain in the reduced dimensions. A similar concept has been theoretically proposed for the nonlinear spin Hall effect in strained graphene and Dirac semimetal [13,42]. We expect future theoretical studies will clarify how to effectively design symmetry breaking to induce a large nonlinear spin-charge conversion.

We acknowledge A. Kurita for the technical assistance of thin film growth, Y. Toyooka for the synthesis of the target, and J. Uzuhashi for transmission electron microscopy. This work was supported by Grants-in-Aid for Scientific Research Grants No. 19H02604 (Y. K.), 19K04499 (S. I.), 20K1482 (K. M.), and 18H01171 (J. F.). This work was partly carried out at NIMS Nanofabrication Platform and NIMS Namiki Foundry.

*Corresponding author. KOZUKA.Yusuke@nims.go.jp

[1] J. Sinova, S. O. Valenzuela, J. Wunderlich, C. H. Back, and T. Jungwirth, Rev. Mod. Phys. 87, 1213 (2015).

[2] S. Murakami, N. Nagaosa, and S.-C. Zhang, Science 301, 1348 (2003).
[3] I. M. Miron, K. Garello, G. Gaudin, P.-J. Zermatten, M. V. Costache, S. Auffret, S. Bandiera, B. Rodmacq, A. Schuhl, and P. Gambardella, Nature (London) 476, 189 (2011).

[4] R. Ramaswamy, J. M. Lee, K. Cai, and H. Yang, Appl. Phys. Rev. 5, 031107 (2018).

[5] A. Manchon, J. Železný, I. M. Miron, T. Jungwirth, J. Sinova, A. Thiaville, K. Garello, and P. Gambardella, Rev. Mod. Phys. 91, 035004 (2019).

[6] J. E. Hirsch, Phys. Rev. Lett. 83, 1834 (1999).

[7] M. Kohmoto, Ann. Phys. (N.Y.) 160, 343 (1985).

[8] Z. Fang, N. Nagaosa, K. S. Takahashi, A. Asamitsu, R. Mathieu, T. Ogasawara, H. Yamada, M. Kawasaki, Y. Tokura, and K. Terakura, Science 302, 92 (2003).

[9] I. Sodemann and L. Fu, Phys. Rev. Lett. 115, 216806 (2015).

[10] Y. Zhang, Y. Sun, and B. Yan, Phys. Rev. B 97, 041101(R) (2018).

[11] J.-S. You, S. Fang, S.-Y. Xu, E. Kaxiras, and T. Low, Phys. Rev. B 98, 121109(R) (2018).

[12] Z. Z. Du, C. M. Wang, H.-Z. Lu, and X. C. Xie, Phys. Rev. Lett. 121, 266601 (2018).

[13] R. Battilomo, N. Scopigno, and C. Ortix, Phys. Rev. Lett. 123, 196403 (2019).

[14] Q. Ma et al., Nature (London) 565, 337 (2019).

[15] K. Kang, T. Li, E. Sohn, J. Shan, and K. F. Mak, Nat. Mater. 18, 324 (2019).

[16] P. He, S. S.-L. Zhang, D. Zhu, Y. Liu, Y. Wang, J. Yu, G. Vignale, and H. Yang, Nat. Phys. 14, 495 (2018).

[17] P. He, S. S.-L. Zhang, D. Zhu, S. Shi, O. G. Heinonen, G. Vignale, and H. Yang, Phys. Rev. Lett. 123, 016801 (2019).

[18] H. Zhang, K. Haule, and D. Vanderbilt, Phys. Rev. Lett. 111, 246402 (2013).

[19] Y. F. Nie, P. D. C. King, C. H. Kim, M. Uchida, H. I. Wei, B. D. Faeth, J. P. Ruf, J. P. C. Ruff, L. Xie, X. Pan, C. J. Fennie, D. G. Schlom, and K. M. Shen, Phys. Rev. Lett. 114, 016401 (2015).

[20] Z. T. Liu, M. Y. Li, Q. F. Li, J. S. Liu, W. Li, H. F. Yang, Q. Yao, C. C. Fan, X. G. Wan, Z. Wang, and D. W. Shen, Sci. Rep. 6, 30309 (2016).

[21] J. Fujioka, T. Okawa, A. Yamamoto, and Y. Tokura, Phys. Rev. B 95, 121102(R) (2017).

[22] A. S. Patri, K. Hwang, H.-W. Lee, and Y. B. Kim, Sci. Rep. 8, 8052 (2018).

[23] H. Wang, K.-Y. Meng, P. Zhang, J. T. Hou, J. Finley, J. Han, F. Yang, and L. Liu, Appl. Phys. Lett. 114, 232406 (2019).

[24] A. S. Everhardt, M. DC, X. Huang, S. Sayed, T. A. Gosavi, Y. Tang, C.-C. Lin, S. Manipatruni, I. A. Young, S. Datta, J.-P. Wang, and R. Ramesh, Phys. Rev. Mater. 3, 051201(R) (2019).

[25] T. Nan, T. J. Anderson, J. Gibbons, K. Hwang, N. Campbell, H. Zhou, Y. Q. Dong, G. Y. Kim, D. F. Shao, T. R. Paudel, N. Reynolds, X. J. Wang, N. X. Sun, E. Y. Tsymbal, S. Y. Choi, M. S. Rzchowski, Y. B. Kim, D. C. Ralph, and C. B. Eom, Proc. Natl. Acad. Sci. U.S.A. 116, 16186 (2019).

[26] L. Liu, Q. Qin, W. Lin, C. Li, Q. Xie, S. He, X. Shu, C. Zhou, Z. Lim, J. Yu, W. Lu, M. Li, X. Yan, S. J. Pennycook, and J. Chen, Nat. Nanotechnol. 14, 939 (2019).

[27] B. L. Chamberland and A. R. Philpotts, J. Alloys Compd. 182, 355 (1992). 
[28] See Supplemental Material at http://link.aps.org/ supplemental/10.1103/PhysRevLett.126.236801 for the details of structural and transport properties, and firstprinciples calculation.

[29] K. Momma and F. Izumi, J. Appl. Crystallogr. 44, 1272 (2011).

[30] G. Kresse and J. Hafner, Phys. Rev. B 47, 558 (1993); G. Kresse and J. Furthmuller, Comput. Mater. Sci. 6, 15 (1996); Phys. Rev. B 54, 11169 (1996).

[31] J. P. Perdew, K. Burke, and M. Ernzerhof, Phys. Rev. Lett. 77, 3865 (1996); 78, 1396(E) (1997).

[32] P. E. Blöchl, Phys. Rev. B 50, 17953 (1994); G. Kresse and D. Joubert, Phys. Rev. B 59, 1758 (1999).

[33] L. Zhang, Q. Liang, Y. Xiong, B. Zhang, L. Gao, H. Li, Y. B. Chen, J. Zhou, S.-T. Zhang, Z.-B. Gu, S. Yao, Z. Wang, Y. Lin, and Y.-F. Chen, Phys. Rev. B 91, 035110 (2015); 92, 039904(E) (2015).

[34] L. Fruchter, O. Schneegans, and Z. Z. Li, J. Appl. Phys. 120, 075307 (2016).
[35] D. J. Groenendijk, C. Autieri, J. Girovsky, M. C. MartinezVelarte, N. Manca, G. Mattoni, A. M. R. V. L. Monteiro, N. Gauquelin, J. Verbeeck, A. F. Otte, M. Gabay, S. Picozzi, and A. D. Caviglia, Phys. Rev. Lett. 119, 256403 (2017).

[36] Y. Nakamura and Y. Yanase, J. Phys. Soc. Jpn. 82, 083705 (2013).

[37] P. D. C. King, S. McKeown Walker, A. Tamai, A. de la Torre, T. Eknapakul, P. Buaphet, S.-K. Mo, W. Meevasana, M. S. Bahramy, and F. Baumberger, Nat. Commun. 5, 3414 (2014).

[38] K. R. Kleindienst, K. Wolff, J. Schubert, R. Schneider, and D. Fuchs, Phys. Rev. B 98, 115113 (2018).

[39] J. Liu, D. Kriegner, L. Horak, D. Puggioni, C. Rayan Serrao, R. Chen, D. Yi, C. Frontera, V. Holy, A. Vishwanath, J. M. Rondinelli, X. Marti, and R. Ramesh, Phys. Rev. B 93, 085118 (2016).

[40] S. Nandy and I. Sodemann, Phys. Rev. B 100, 195117 (2019).

[41] Z. Z. Du, C. M. Wang, S. Li, H.-Z. Lu, and X. C. Xie, Nat. Commun. 10, 3047 (2019).

[42] Y. Araki, Sci. Rep. 8, 15236 (2018). 